\title{
Analysis of Active Power Flow Control with Phase Shifting Transformer in AC Transmission Line
}

\author{
Thandar $\mathrm{Oo}^{1}$, Su Mon Myint ${ }^{2}$, Aye Khaing $\mathrm{Zin}^{3}$ \\ ${ }^{1}$ ME Student, ${ }^{2}$ Lecturer, ${ }^{3}$ Assistant Lecturer \\ Electrical Power Department, Pyay Technological University \\ The Republic of the Union of Myanmar, Pyay, Myanmar
}

\begin{abstract}
Nowadays, active power flow should be controlled because voltage fluctuation occurred on various load conditions. Phase shifting transformer (PST) is used to control the active power flow in three phase electricity transmission network. It can increase reliability and can serve as an alternative to new investments in overhead lines. The control of active power flow is achieved by adjusting the phase angle of the voltages at the PST terminals. In this paper, 230 $\mathrm{kV} \mathrm{AC}$ transmission line is chosen for active power flow control. Two conditions, maximum and minimum load conditions are considered as a case study for active power flow control performance in a selected line. Simulation of active power flow with PST model is carried out by using Matlab software. Finally, according to simulation results, system voltages and active power depend phase angles are revealed in this paper.
\end{abstract}

Keywords: phase shifting transformer, on load tap changer, transmission line, phase shift angle, active power flow control

\section{INTRODUCTION}

Electric power is transported between different voltage levels of the electric grid through interconnected transmission lines. Overloading in transmission line decreases the security of power system. When power flows between two nodes, there is a voltage drop and a phase angle different between the sending end and receiving end. So, the power flow needs to control in order to achieve the optimal utilization of transmission lines capacity. Operating efficiency of transmission can be improved by using FACTS device. Phase Shifting Transformer is one of the FACTS families which can be used for power

control in a network. Therefore, the phase-shifting transformer is the first choice for controlling the active power flow between two system areas as it is the less expensive than the FACTS devices, and performs satisfactorily under steady-state conditions. For example, the PST can be used in order to impose a power flow according to the contractual amount. For this reason, the PSTs are usually installed on interconnection lines [1].

In interconnected power networks, certain ac transmission lines do not have definite sender and receiver ends since the active power conveyed by these lines does not flow in the same direction at all times. The polarity of phase shift $\delta$ between the voltages at two regions of an interconnected power network is very important because it determines the direction of the active power flow in the corresponding ac transmission line [4]. PST can alter the polarity of phase angle $\delta$ between the voltages by using OLTC. PSTs compensates for the drop by inserting, between its sending and its receiving ends, a series voltage in quadrature with the line-to-ground voltages. This quadrature voltage produces, between the PSTs terminals, a phase shift whose magnitude varies with the magnitude of the quadrature voltage [3].

The purpose of PST is usually the control of power flow over transmission lines. Both the magnitude and the direction of the power flow can be controlled by varying the phase shift. Although PSTs enable the control of power flowing through a line, the flow itself is still a function of the grid situation, and the power flow can only be "shift" [9]. 
II. POWER FLOW CONTROL

A. Controlling the power flow through a transmission line

A simplified relation between active power transported over a transmission line between sending and receiving nodes and the complex voltages at both nodes is:

$\mathrm{P}_{\text {Line }}=\frac{\left|\mathrm{V}_{\mathrm{S}}\right|\left|\mathrm{V}_{\mathrm{R}}\right|}{\mathrm{X}_{\text {Line }}} \sin (\delta)$

Where sending node $V_{S}$ and receiving node $V_{R}, \delta$ the phase angle between these voltages and $X_{\text {Line }}$ the impedance. The active power is proportional to the voltages on the sending and the receiving ends and the phase angle between both ends; it is also inversely proportional to the line reactance. Therefore, the power flow through the line can be controlled by altering the voltage at a node, the impedance between nodes and the angle between terminal voltages. The line flow can also be altered by changing the line impedance. This can be done by inserting variable series impedance. Then, the active power flow can also be control by altering the phase angle between sending and receiving ends voltage with a series injected voltage in quadrature to the phase voltage.

\section{B. Phase Shifting Transformer}

The PST is installed as a series reactance with a phase shift in transmission line. The power flow through the line is increased by adding an angle $\alpha$ to the existing angle $\delta$. The phase shift is controllable within certain limits. The PST is a mechanically switched device which inserts a transformed, variable (using a tap changer) line voltage in series with the phase voltage. Figure 1(a) depicts this for direct symmetrical phaseshifting transformer and Figure 1(b) depicts its phasor diagram.

$\mathrm{P}_{\text {Line }}=\frac{\left|\mathrm{V}_{\mathrm{S}}\right|\left|\mathrm{V}_{\mathrm{R}}\right|}{\mathrm{X}_{\text {Line }}+\mathrm{X}_{\text {PST }}} \sin (\delta+\alpha)$

The state above can also be interpreted in another way: the active power is proportional to the phase angle between nodes. Altering the active power can be done by altering the phase angles. Although phase shifting transformers enable the control of power flowing through a line, the flow itself is still a function of the grid situation, and the power flow can only be "shifted" [9].

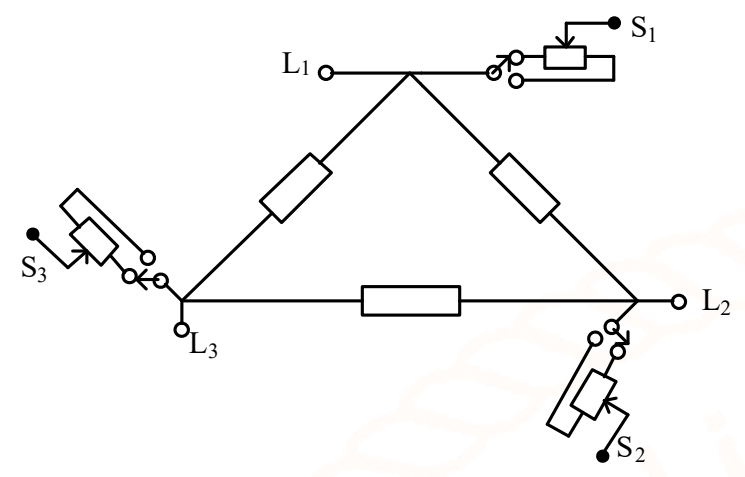

(a)

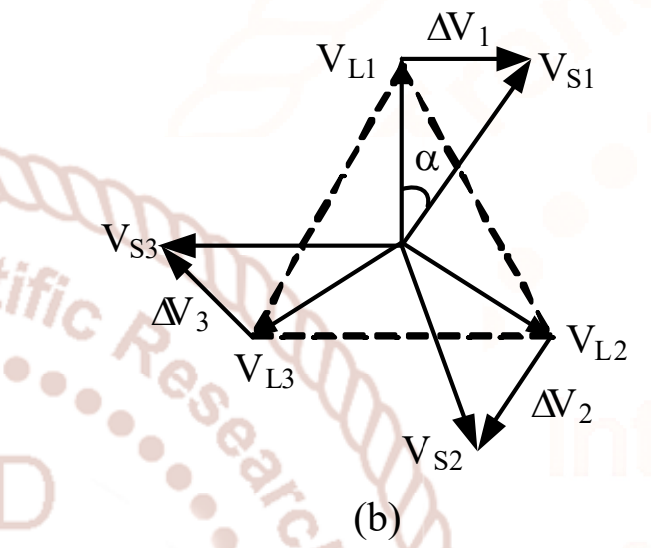

Figure.1. Direct symmetrical PST (a) Circuit diagram and (b) Phasor diagram

III. PRINCIPLES OF PHASE SHIFTING TRANSFORMER

\section{A. Basic Theory of Phase Shifting Transformer}

The phase shifting transformer (PST) is a specialized form of transformer used to control the active power flow in three phase electricity transmission networks. The term Phase Shifter is more generally used to indicate a device which can inject a voltage with a controllable phase angle and/or magnitude under noload (off-load) and load (on-load) conditions.

Operation of a conventional phase-shifter is characterized by:

$>$ High response time as a result of inertia of moving parts, and

$>$ High level of maintenance due to mechanical contacts and oil deterioration.

The main power circuitry of the phase shifting transformer consists of Figure 2(a): the exciting transformer that provides input voltage to the phase shifter; the series transformer, that injects a controllable voltage $\mathrm{V}_{\alpha}$ in the transmission line; and the converter or tap changer, which controls the magnitude and/or phase angle of the injected voltage. A converter is used in case of a power electronic based interface, and a tap changer is used in case of a 
mechanical controlled phase shifting transformer.

The PST is a device that is used for power flow control in order to relieve congestions in electrical grids. Figure 2(a) shows a schematic diagram of a phase shifter installed on a transmission line between buses $a$ and $b$. The sending and receiving ends of the transmission line are represented by voltage phasors $\mathrm{V}_{\mathrm{S}}$ and $\mathrm{V}_{\mathrm{R}}$, and corresponding impedances $\mathrm{Z}_{\mathrm{S}}$ and $\mathrm{Z}_{\mathrm{R}}$, respectively.

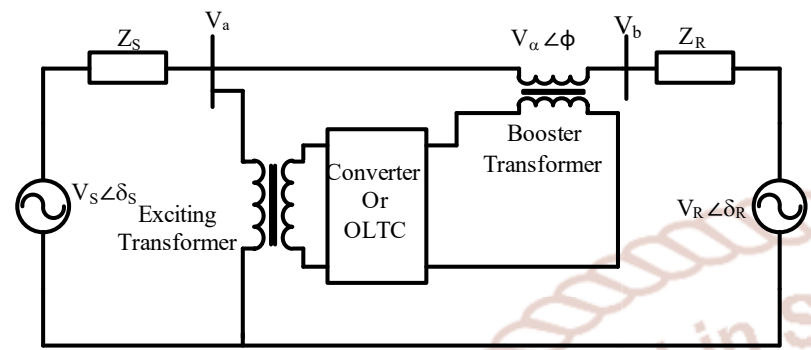

(a)

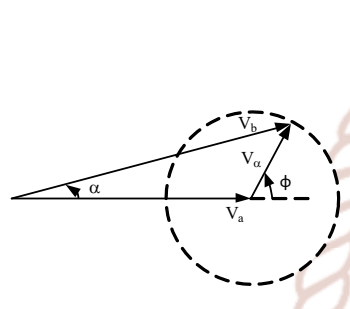

(b)

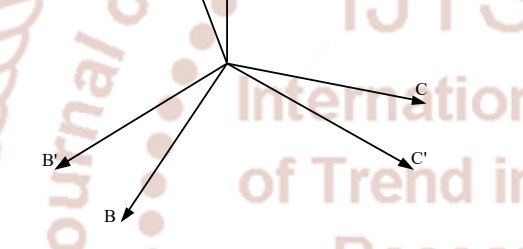

(c)

Figure.2. Diagrams of a phase-shifting transformer:

(a) Schematic diagram; (b) Phasor diagram; (c) Phase shift for advance [1]

Depending on the magnitude and phase angle of the injected voltage $\mathrm{V}_{\alpha}$, the magnitude and/or phase-angle of the system voltage $V_{b}$ is varied Figure 2(b). With a flexible phase shifting transformer, the control range achieved is a circle with centre in the tip of the phasor $\mathrm{V}_{\mathrm{a}}$ and radius equal to the amplitude of $\mathrm{V}_{\alpha}$. The output voltage of the phase shifting transformer is controlled by varying the amplitude and angle of the phasor $\mathrm{V}_{\alpha}$, that is $\mathrm{V}_{\alpha}$ and $\varphi[1]$.

The active power flow on the transmission line that incorporates a PST is given by:

$$
\mathrm{P}=\frac{\left|\mathrm{V}_{\mathrm{S}}\right|\left|\mathrm{V}_{\mathrm{R}}\right|}{\mathrm{X}_{\text {eq }}} \sin \left(\delta_{S}-\delta_{\mathrm{R}} \pm \alpha\right)
$$

Where $\mathrm{X}_{\mathrm{eq}}$ is the net equivalent reactance of the line and the sources, whereas $\delta_{\mathrm{S}}$ and $\delta_{\mathrm{R}}$ are phase angles of the phasors $\mathrm{V}_{\mathrm{S}}$ and $\mathrm{V}_{\mathrm{R}}$, respectively. Based on equation (3) the angle $\alpha$ is the dominant variable for power flow control.
B. Categories of Phase Shifting Transformer

PST's come in many different forms. They can be classified by these characteristics:

$>$ Direct PST's are based on one three-phase core. The phase shift is obtained by connecting the windings in an appropriate manner.

$>$ Indirect PST's are based on a construction with two separate transformers; one variable tap exciter to regulate the amplitude of the quadrature voltage and one series transformer to inject the quadrature voltage in the right phase.

$>$ Asymmetrical PST's create an output voltage with an altered phase angle and amplitude compared to the input voltage.

Symmetrical PST's create an output voltage with an altered phase angle compared to the input voltage, but with the same amplitude [7].

\section{Delta-hexagonal Type Phase Shifting Transformer}

In this paper, Delta-hexagonal type Phase Shifting Transformer is applied for active power flow control. This type of PST can be symmetrical with two tap changers, as shown in Figure 3, or non-symmetrical with only one tap changer. To maintain symmetry when two tap changers are used, the tap changers are often interlocked such that they take alternating steps from neutral to maintain no more than one step off of symmetry.

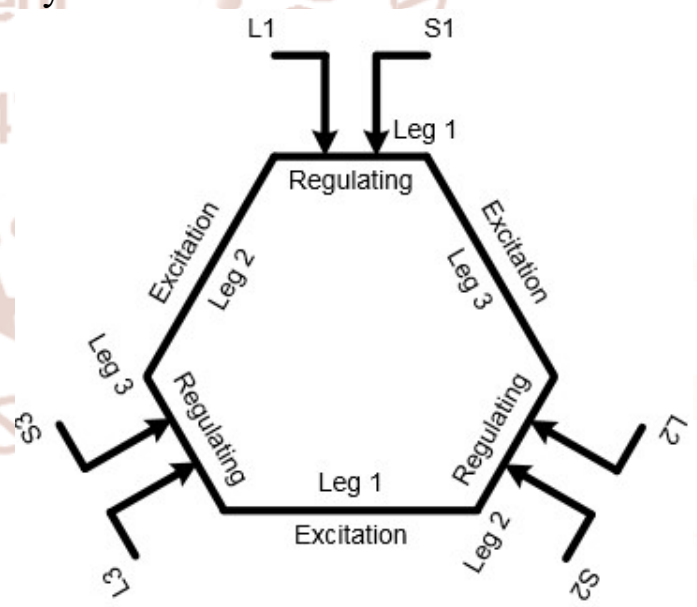

Figure.3. Delta-hexagonal type phase shifting transformer

An important advantage of this design is that only one tap variable transformer is need per phase. Deltahexagonal design PST has further advantage that the phase angle changes almost linearly with tap-changer setting. It is often used for lower voltage level. 


\section{CASE STUDY}

$230 \mathrm{kV}$ Toungoo-Tharyagone line is selected for active power flow control by using PST. This line is important since it is one of a power feeding line to lower Myanmar National Grid. This transmission line is connected to Kamarnet-Hlawga line that is one of a power carrying transmission line to Yangon City. The active power flow control becomes importance for the selected line because the power demand is varied on every time. For the control of active power flow, PST is installed in series with the selected line. Line data and line parameter of Toungoo-Tharyagone transmission line are shown in Table 1 and Table 2.

The parameters of PST are shown in Table 3. In this case 200 MVA throughput power of PST is chosen depend on the maximum load condition in the selected line. For the active power flow performance 16 steps On-load Tap Changer and \pm 30 degrees phase shift are selected. Thus, the tap is change from -8 to 8 and the phase angle change for each tap is 3.75 degrees.

Table1. Line Data of $230 \mathrm{kV}$ Toungoo-Tharyagone Line

\begin{tabular}{|c|c|c|c|}
\hline Months & $\begin{array}{c}\mathbf{P}_{\max } \\
(\mathbf{M W})\end{array}$ & $\begin{array}{c}\mathbf{Q}_{\max } \\
(\text { MVAr) }\end{array}$ & $\begin{array}{c}\mathbf{S}_{\max } \\
\text { (MVA) }\end{array}$ \\
\hline April(2017) & 263.57 & -15.54 & 264.03 \\
\hline July (2017) & 274.92 & 3.62 & 274.94 \\
\hline December(2017) & 290.95 & 9.80 & 291.11 \\
\hline
\end{tabular}




\section{B. Simulink Model without Phase Shifting Transformer}

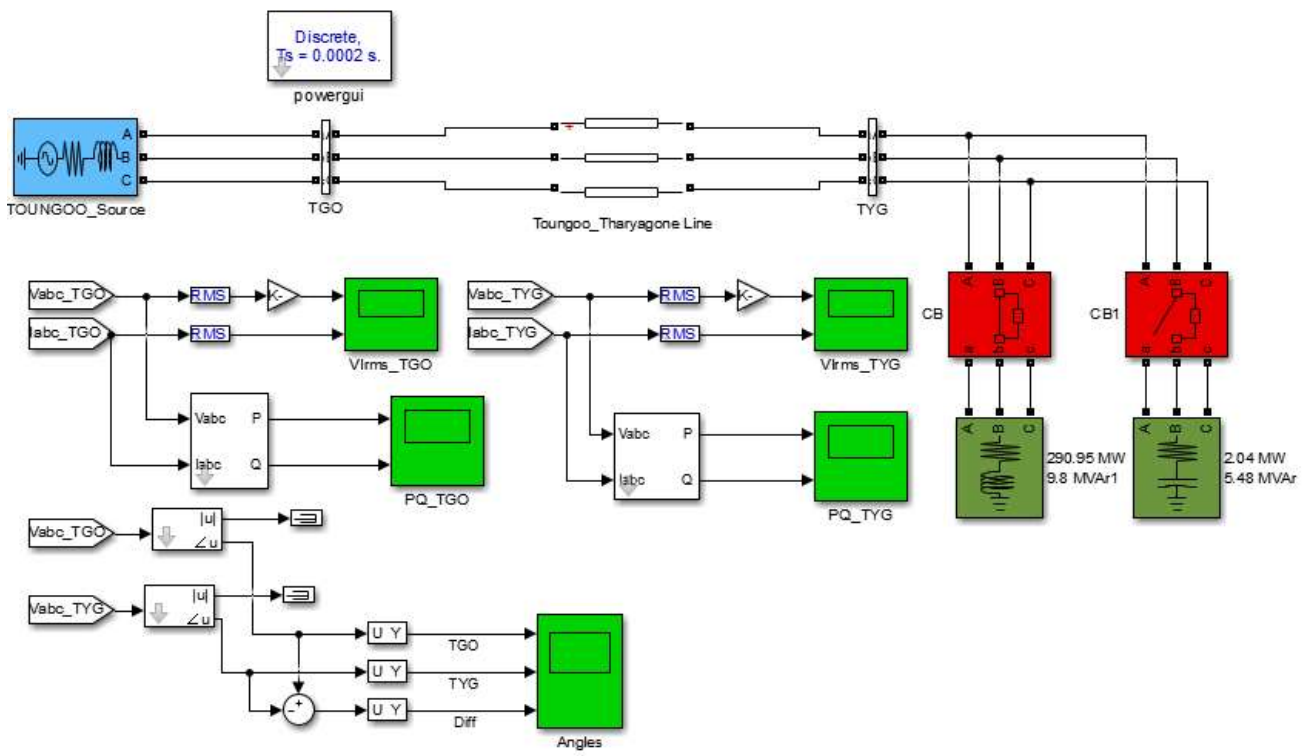

Figure.5. Simulink model of without PST under load condition

Figure 5 shows the Simulink model of without PST under maximum and minimum load conditions in 230 $\mathrm{kV}$ Toungoo-Tharyagone line. Before using PST, the active power flow on line is $264 \mathrm{MW}$ from simulation the results.

\section{Simulations Results of Case Study}

The paper presents the analysis for active power flow control performance under maximum and minimum load conditions without and with PST in selected transmission line. From simulation results, the comparison under maximum and minimum load conditions for phase angle difference between the voltages with related to tap positions, active power flow with related to tap positions, receiving end voltage with related to tap positions, and phase difference with related to power flow is shown in Figure 6, Figure 7, Figure 8 and Figure 9.

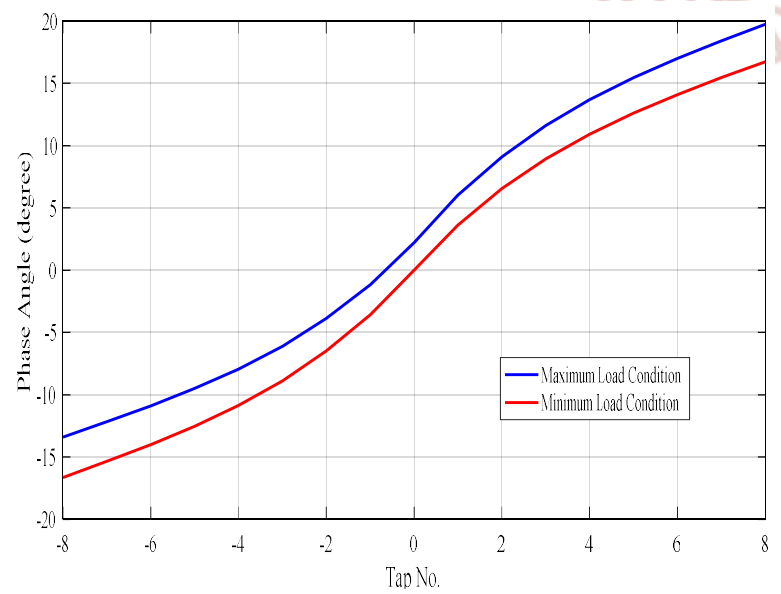

Figure.6. Comparison of result under maximum and minimum load conditions for the phase angle with related to tap positions
Figure 6 presents the comparison of the phase angle with related to tap positions from simulation results. In this figure, the phase angle on minimum load condition is lower than the maximum load condition. The phase angle is also increased by raising the tap positions.

In Figure 7, the active power flow on the line is different on various load conditions. From this result, it is clear that the active power flow on line is controlled by changing the tap positions.

Figure 8 shows the comparison result for the receiving end voltage with related to tap positions from simulation results. In this figure, the receiving end voltage for maximum load condition is lower than the minimum load condition. The receiving end voltage decreased due to the load growth. When the tap positions increased, the receiving end voltage decreased.

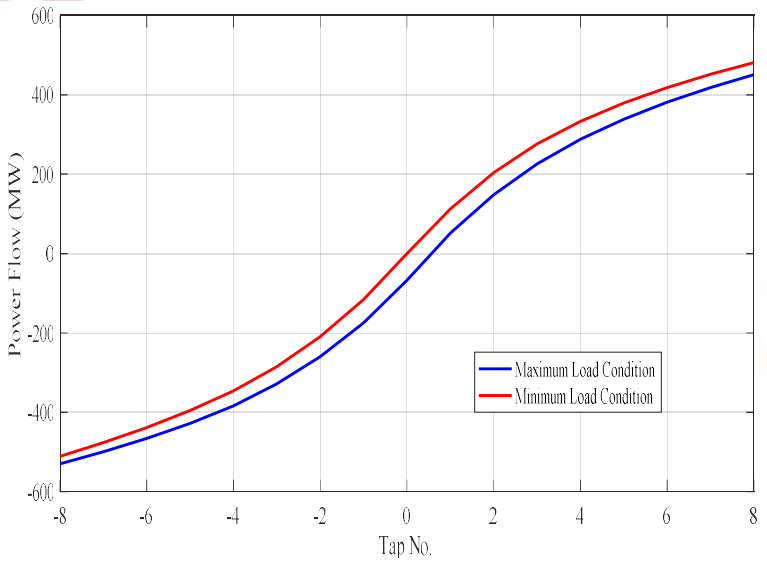

Figure.7. Comparison of active power flow with related to tap positions under maximum and minimum load conditions 


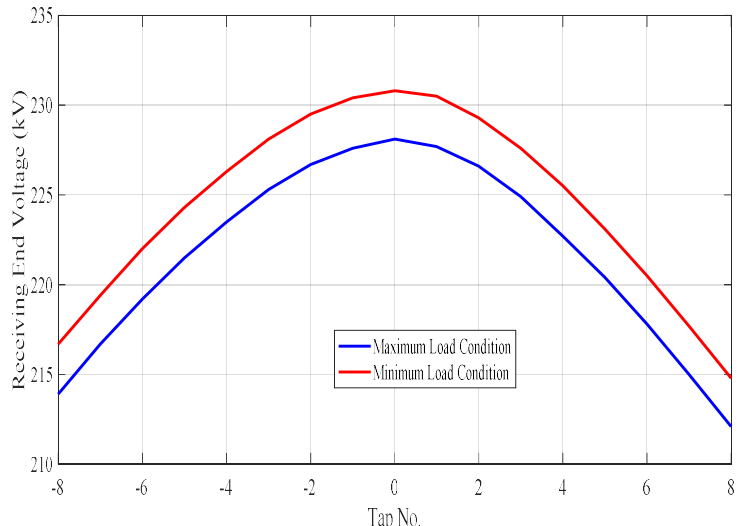

Figure.8. Comparison of result under maximum and minimum load conditions for the receiving end voltage with related to tap positions

The comparison of the phase angle with related to active power flow from simulation result is illustrated in Figure 9. From the results, the phase angle on maximum load condition is larger than the minimum load condition and the improvement of active power flow on the line depends on the phase angle increasing in phase shifting transformer.

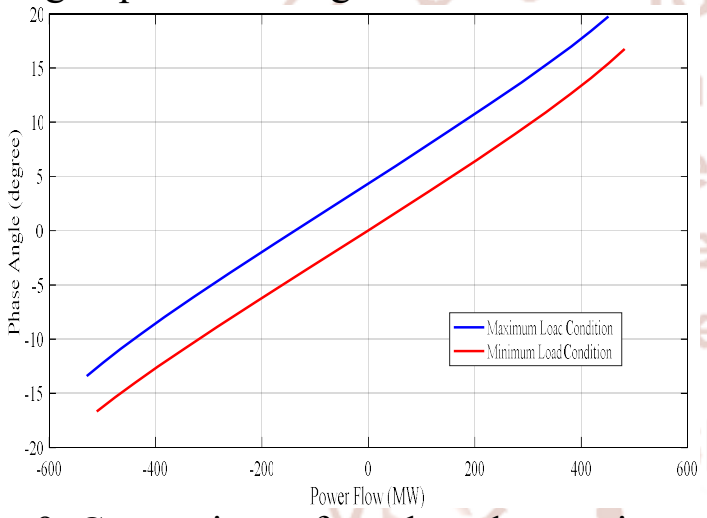

Figure.9. Comparison of result under maximum and minimum load conditions for the phase angle with related to active power flow

\section{CONCLUSION}

The active power flow on the line is increased by controlling the phase angles between sending ends and receiving end voltages. The phase angles are controlled by changing the tap position on the OLTC. In this paper, Direct Symmetrical Delta-hexagonal type PST is chosen for the connections of transformer. Matlab software is applied for the simulation results. Two models for active power flow control with and without PST under load condition are described. In the result, before using PST the active power flow on line for maximum load condition is $264 \mathrm{MW}$ and for minimum load condition is 2.26 MW. By using PST, the active power flow on the line is controlled from 67.27 MW to 450.89 MW for maximum load condition and $-0.84 \mathrm{MW}$ to $481.52 \mathrm{MW}$ for minimum load condition. So, PST can control an active power flow in transmission lines.

\section{ACKNOWLEDGEMENTS}

First at all, the author would like to express her special thanks to Dr. Nyaunt Soe, Rector, Pyay Technological University for initiating the Master programme at the Pyay Technological University. The author is sincerely grateful to her teacher Dr. Soe Winn, Professor and Head, Department of Electrical Power Engineering, Pyay Technological University, for his patience, permissions and helpful suggestions. The author is deeply thankful to her supervisor, Dr. $\mathrm{Su}$ Mon Myint, Lecturer for her guidance, valuable advice and her co-supervisor, Daw Aye Khaing Zin, Assistant Lecturer for her worthy advice at Department of Electrical Power Engineering, Pyay Technological University. And, the author is deeply grateful to her teacher U Than Zaw Htwe, Lecturer, Department of Electrical Power Engineering, Yangon Technological University, for his valuable instructions. My heart-felt thanks to my family, friends and colleagues who have me for the completion of this paper.

\section{REFERENCES}

1. Dorian O. SIDEA, Lucian TOMA, Mircea EREMIA; "Sizing a phase shifting transformer for congestion management in high wind generation areas", Department of Electrical Power Systems, University Politehnica of Bucharest, Bucharest, Romania: June 2017.

2. Michael Thompson; "Protection System for Phase-Shifting Transformers Improves Simplicity, Dependability, and Security", Schweitzer Engineering Laboratories, Inc: Revised edition released May 2015.

3. J. M. Guzman, F. J. Orega-Herrera, J. TorresJimenez, G. Tapia-Tinoco, L.A. ContrerasAugilar, "Implementation of Phase-Shifting Transformer model into an OPF formulation by Matlab optimization toolbox", International Journal of Scientific and Research Publications, Volume 3, Issue 9, September 2013, ISSN 22503153.

4. FESTO, Electricity and New Energy, Lab Volt Series, "AC Transmission Lines", 120V-60Hz

5. Hingorani, N. G., Gyugyi, L; Understanding FACTS. New Youk: IEEE Press, 2000,432 s. 
6. James H. Harlow. p. cm.-( The Electrical Power Engineering Series; 9) Includes bibliographical references and index. ISBN 0-8493-1704-5 (alk.paper). 1. Electric transformers. I . Harlow, James H. II. title. III Series.

7. Verboomen, J., Hertem, D. V., Schavemaker, P. H., Kling, W. L. and Belmans, R.: Phase Shifting Transformers: Principles and Applications, IEEE Conf. on Future Power Systems, Amsterdam, November (2005)
8. Jozef RUSNAK; POWER FLOW CONTROL BY USE OF PHASE-SHIFTING TRANSFORMER, Ph.D. Degree Programme, Dept. of Electric Power Engineering, FEI TU, Kosice.

9. Dirk Van Hertem*; Influence of phase shifting transformers and HVDC on power system losses. Graduate Student Menber, IEEE, Jody Verboomen, Member, IEEE, Stijn Cole*, Graduate Student Member, IEEE, Wil Kling, Member, IEEE and Ronnie Belmans, Fellow, IEEE *research group ELECTA, electrical engineering department, KU Leuven, Belgium.

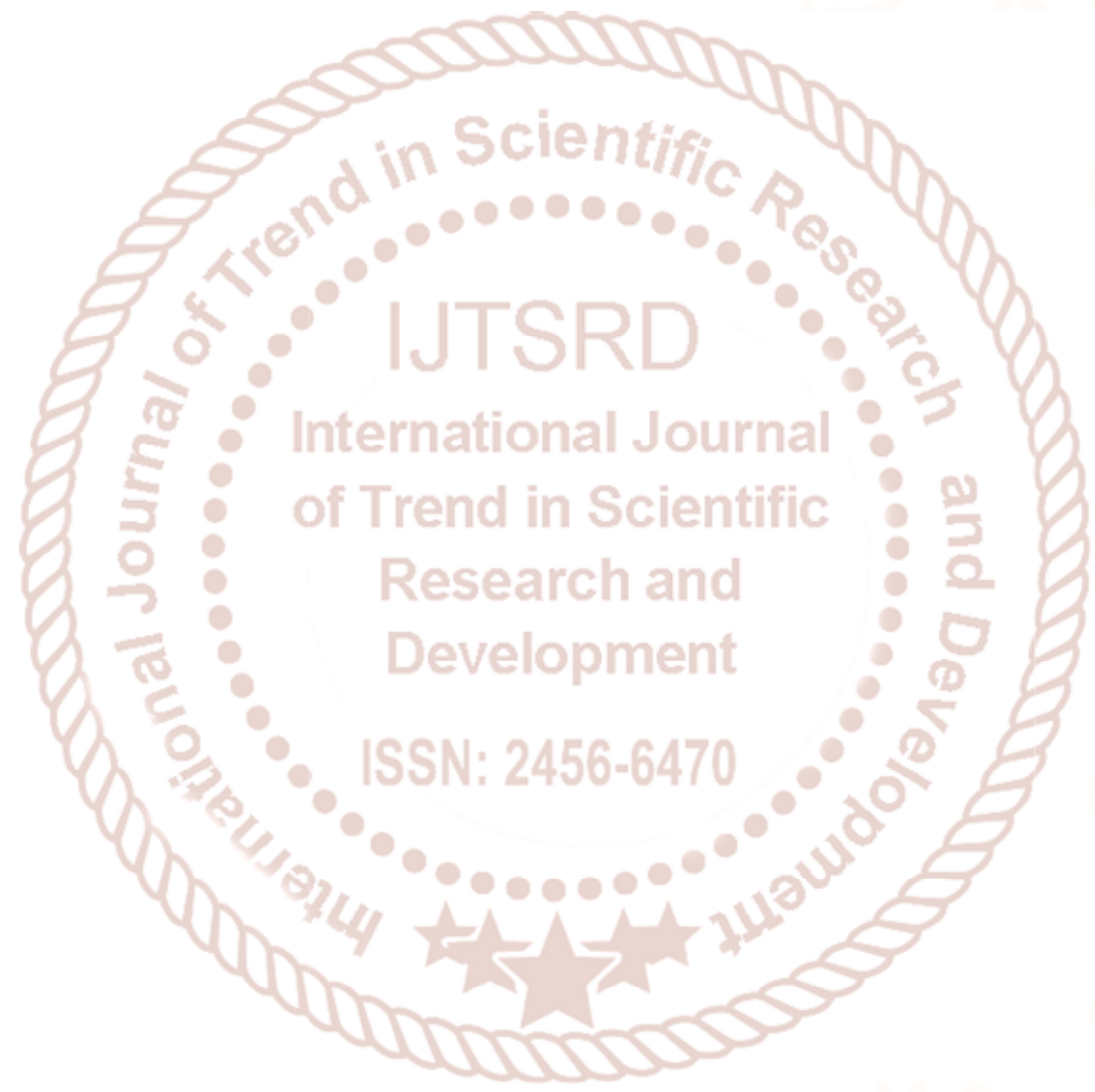

